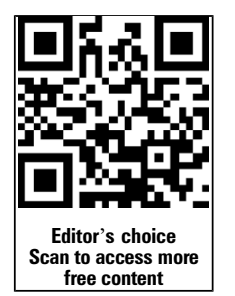

free content

${ }^{1}$ School of Public Health, Peking University, Beijing, China

2 Peking University Eye Center, Peking University Third Hospital, Beijing, China

Correspondence to Dr Xiao-Ming Lin, School of Public Health, Peking University, 38 Xueyuan Road, Beijing 100191, China; linbjmu@bjmu.edu.cn

Received 6 May 2014 Revised 24 August 2014 Accepted 28 August 2014 Published Online First 16 September 2014

\title{
Changes following supplementation with lutein and zeaxanthin in retinal function in eyes with early age-related macular degeneration: a randomised, double-blind, placebo-controlled trial
}

\author{
Yang-Mu Huang, ${ }^{1}$ Hong-Liang Dou, ${ }^{2}$ Fei-Fei Huang, ${ }^{1}$ Xian-Rong $\mathrm{Xu}{ }^{1}$ \\ Zhi-Yong Zou, ${ }^{1}$ Xin-Rong Lu, ${ }^{2}$ Xiao-Ming Lin ${ }^{1}$
}

\begin{abstract}
Aims To investigate functional and macular pigment (MP) changes in patients with early age-related macular degeneration (AMD) after multiple supplementation with lutein and zeaxanthin.

Methods 112 patients with early AMD were randomly (1:1:1:1) assigned to receive $10 \mathrm{mg}$ lutein, $20 \mathrm{mg}$ lutein, lutein $(10 \mathrm{mg})+$ zeaxanthin $(10 \mathrm{mg})$, or placebo daily for 2 years. MP optical density (MPOD) was recorded at baseline, 48 weeks and 2 years. Retinal sensitivities were measured by multifocal electroretinogram for peak-totrough amplitude (N1P1) at baseline and at 48 weeks, and in terms of microperimeter-determined mean retinal sensitivity (MRS) at 48 weeks and 2 years.

Results Supplementation with lutein and zeaxanthin augmented MPOD significantly in active treatment groups (all $p<0.05$ ). N1P1 response densities showed significant increases in ring 1 and ring 2 after 48 weeks of supplementation, while no significant changes were seen in rings $3-6$. Significant increases in MRS were detected after supplementation with either 10 or $20 \mathrm{mg}$ lutein, whereas no such increases were seen in the placebo arm.

Conclusions Supplementation with lutein and/or zeaxanthin increases MPOD, and supplemental lutein enhances retinal sensitivity, in patients with early AMD. Trial registration number Clinicaltrials.gov NCT10528605.
\end{abstract}

\section{INTRODUCTION}

Age-related macular degeneration (AMD) is a chronic, progressive, degenerative eye disease affecting the central cone-rich region of the retina responsible for highest visual acuity. ${ }^{1}{ }^{2}$ It has become the major cause of legal blindness in the elderly, in both developed and developing countries. ${ }^{3}$ It has been shown that oxidative stress related to low-grade inflammation and hypoxia in the outer retina are important in the pathogenesis of AMD. ${ }^{1}$ This is consistent with the idea that low macular pigment optical density (MPOD) might be a risk factor for $\mathrm{AMD}$, because the components of macular pigment (MP), the macular carotenoids (lutein, zeaxanthin and meso-zeaxanthin), show potent properties as antioxidants. ${ }^{4}$

Indeed, many large observational studies have shown an inverse relationship between the dietary intake of lutein/zeaxanthin and risk of AMD..$^{5}$ Data from intervention studies also suggest that supplementation with macular carotenoids may increase MPOD and improve visual function in patients with AMD. ${ }^{7-9}$ However, some AMD trials have found that, although MPOD increased after lutein supplementation, visual function did not show significant improvements. ${ }^{10}{ }^{11}$ This may be because significant morphological changes do not adversely affect retinal function at the earlier stage, leaving little room for measurable improvement. ${ }^{12} 13$ This supports the notion that early intervention might be more effective in enhancing or maintaining visual function. ${ }^{14}$ Also, limited use of sensitive visual measurements, such as microperimeter (MP-1) and multifocal electroretinogram (mfERG), the two objective and appropriately localised approaches, may have precluded identification of clinically important visual benefits of supplementation with macular carotenoids. ${ }^{8} 13$

The above explanations prompted this study, which is designed to investigate the effects of supplementation with macular carotenoids on early AMD using more sensitive measurements. Unfortunately, few studies have focused on early AMD, or used both mfERG and MP-1 to evaluate retinal function after supplementation. Further, although zeaxanthin and meso-zeaxanthin are the dominant carotenoids in the central macula, few studies have reported on supplementation with high doses of zeaxanthin in combination with lutein. ${ }^{7}$ Thus, the purpose of this study was to determine the effect of lutein/zeaxanthin supplementation on MPOD and retinal functions, and how increasing MPOD might affect retinal sensitivities measured by MP-1 and mfERG.

\section{MATERIALS AND METHODS}

In this randomised, double-blind, placebocontrolled trial, 112 patients with early AMD from Beijing, China were investigated. Each subject was screened, and had a clinical diagnosis of early AMD (defined as the presence of soft drusen and/ or retinal pigmentary abnormalities, with no signs of late AMD) according to the Age-Related Eye Disease Study System. ${ }^{15}$ Other inclusion criteria were age over 50 years, clear ocular media, and consent to adhere to the study regimen. Those who had other ocular disorders, unstable systemic or chronic illness, or had consumed dietary supplements containing carotenoids within the previous 6 months were excluded. Approval from the medical ethics committee of Peking University was obtained, and the study was performed in 
accordance with the principles of the Declaration of Helsinki. Written informed consent was obtained from all subjects.

Subjects were randomly assigned to take $10 \mathrm{mg}$ lutein, $20 \mathrm{mg}$ lutein, lutein $(10 \mathrm{mg})+$ zeaxanthin $(10 \mathrm{mg})$, or placebo daily for 2 years. The randomisation sequence with stratification by baseline MPOD was computer generated using a permuted block design with block size of 8 . Data on possible risk factors for AMD were collected from baseline examinations, questionnaires on characteristics and demographic data, and a validated foodfrequency questionnaire. MPOD was quantified at baseline, 24 weeks, 48 weeks and 2 years. Retinal sensitivities were measured by mfERG at baseline and 48 weeks, followed by MP- 1 at 48 weeks and 2 years. All ophthalmic examinations were performed by the same technicians from Peking University Eye Center, Peking University Third Hospital. All subjects, examiners and study staff were blinded to treatment assignment, and all capsules were identical in appearance. Subjects were required to maintain their normal dietary and living routines, and to pay a monthly visit to the office to receive capsules for the following month and send back the remaining capsules with a daily check list. Subjects were encouraged to report any adverse effects immediately, and were asked specifically about adverse events such as carotenoderma during visits.

MPOD was measured using fundus autofluorescence images taken with a confocal scanning laser ophthalmoscope (Heidelberg Retina Angiograph II; Heidelberg Engineering, Heidelberg, Germany) centred on the fovea, as detailed elsewhere. ${ }^{16}$ mfERG readings were recorded and analysed with the RETIscan system (V.3.21; Roland Consult, Brandenburg, Germany). Measurements were recorded in keeping with the guidelines of the International Society for Clinical Electrophysiology of Vision as detailed previously. ${ }^{13}$ The mfERG responses were separated into six concentric rings, and the response amplitudes in each ring were measured by N1P1 response densities (amplitudes per unit retinal area in $\mathrm{nV} / \mathrm{deg}^{2}$ ).

Microperimetry was carried out (Microperimeter MP-1; Nidek Technologies, Padova, Italy) with a fundus-controlled device including an eye-tracking system. For assessing visual threshold, a 4-2-1 staircase strategy was used, and a test grid with 41 stimulus loci covering an area of $10^{\circ}$ was applied. The stimuli were projected on to a white background with black illumination set to $1.27 \mathrm{~cd} / \mathrm{m}^{2}$ and a stimulus presentation time of $200 \mathrm{~ms}$. A red $3^{\circ}$ cross was used as the fixation target. After pupil dilatation and dark adaptation for at least $10 \mathrm{~min}$, subjects were asked to press the patient trigger to confirm when they perceived each stimulus throughout the examination. Standard explanations and training were provided before tests. The result was evaluated by mean retinal sensitivity (MRS) as the average sensitivity of the test loci at $1^{\circ}, 3^{\circ}$ and $5^{\circ}$ eccentricities.

\section{Sample size and statistical analyses}

Our primary measure used to estimate sample size was the assumption of a $30 \%$ change in MPOD after treatment. ${ }^{17}$ With a significance level of $5 \%$ and a dropout rate of $10 \%$, we estimated that a sample size of 112 patients was needed to provide $80 \%$ power.

Statistical analyses were conducted using SPSS V.11.0 for Windows software. Baseline comparisons among groups were assessed using analysis of variance (ANOVA) or the $\chi^{2}$ analysis. Skewed data were log-transformed for analysis. Within-group differences during the intervention were tested using paired $t$ tests, and between-group differences at each time point were measured using analysis of covariance. Changes between groups over time were assessed using repeated-measures ANOVA including time $\times$ treatment interaction. The linear correlation between two variables was assessed using the Pearson test. $\mathrm{p}<0.05$ was considered significant.

\section{RESULTS}

A total of 112 subjects were enrolled and randomised after screening of 334 candidates. Four subjects $(3.6 \%)$ were excluded from analysis for failure to attend scheduled visits. Subject characteristics are shown in table 1. Dietary intakes of lutein/ zeaxanthin and other carotenoids were similar among groups, with slight changes during the trial (all $\mathrm{p}>0.05$ ). No adverse events were observed or reported.

MPOD progressively increased after supplementation with lutein/zeaxanthin (all $\mathrm{p}<0.05$ ), while no significant change was observed in the placebo group (table 2). The most significant increase from baseline was detected in the $20 \mathrm{mg}$ lutein group after the first 48 weeks, while, at 2 years, MPOD in the 10 and $20 \mathrm{mg}$ lutein groups were comparable.

As seen in figure 1, N1P1 response densities in ring 1 showed significant within-group increases in all active treatment groups during the first 48 weeks, especially after supplementation with $20 \mathrm{mg}$ lutein $\left(86.8 \mathrm{nV} / \mathrm{deg}^{2}\right)$ and lutein+zeaxanthin $(91.6 \mathrm{nV} /$ $\mathrm{deg}^{2}$ ) (figure 1A), whereas the only significant increase from baseline in ring 2 was detected in the $20 \mathrm{mg}$ lutein group (reflected as an increase of $32.8 \%, \mathrm{p}<0.05$ ) (figure 1B). No significant changes in response densities in rings 3-6 were seen in any group. Repeated-measures analysis showed a significant time effect on improving N1P1 response densities in ring $1(p<0.001)$ and ring $2(p=0.02)$, and a tendency for a treatment effect in ring $1 \quad(p=0.06)$. While the change in N1P1 response densities in ring 1 and ring 2 correlated negatively with baseline MPOD, it correlated positively with the change in MPOD. No significant associations were found between MPOD

Table 1 Baseline characteristics of subjects with early age-related macular degeneration

\begin{tabular}{|c|c|c|c|c|}
\hline Characteristic & $\begin{array}{l}\text { Placebo } \\
(n=28)\end{array}$ & $\begin{array}{l}10 \mathrm{mg} \\
\text { lutein } \\
(n=26)\end{array}$ & $\begin{array}{l}20 \mathrm{mg} \\
\text { lutein } \\
(n=27)\end{array}$ & $\begin{array}{l}\text { lutein } \\
\text { +zeaxanthin } \\
(\mathrm{n}=27)\end{array}$ \\
\hline Age (years) & $69.0 \pm 7.5$ & $69.7 \pm 8.3$ & $69.3 \pm 6.9$ & $68.5 \pm 6.9$ \\
\hline Sex (male/female) & $11 / 17$ & $9 / 17$ & $14 / 13$ & $12 / 15$ \\
\hline Education (years) & $12.2 \pm 2.8$ & $10.8 \pm 2.7$ & $12.2 \pm 2.9$ & $10.5 \pm 4.1$ \\
\hline $\mathrm{BMI}\left(\mathrm{kg} / \mathrm{m}^{2}\right)$ & $24.8 \pm 3.0$ & $24.1 \pm 3.4$ & $25.1 \pm 3.3$ & $24.6 \pm 3.6$ \\
\hline $\begin{array}{l}\text { Waist } \\
\text { circumference }(\mathrm{cm})\end{array}$ & $27.10 \pm 7.61$ & $27.86 \pm 7.25$ & $28.64 \pm 5.93$ & $27.92 \pm 7.19$ \\
\hline \multicolumn{5}{|l|}{ Serum lipids (mmol/L) } \\
\hline Total cholesterol & $5.02 \pm 1.76$ & $4.98 \pm 1.07$ & $5.09 \pm 0.88$ & $5.25 \pm 0.95$ \\
\hline Triglyceride & $1.57 \pm 1.58$ & $1.54 \pm 0.68$ & $1.49 \pm 0.82$ & $1.78 \pm 0.79$ \\
\hline HDL-cholesterol & $1.39 \pm 0.44$ & $1.39 \pm 0.32$ & $1.41 \pm 0.26$ & $1.48 \pm 0.29$ \\
\hline LDL-cholesterol & $3.09 \pm 0.61$ & $3.19 \pm 0.75$ & $3.2 \pm 0.61$ & $3.34 \pm 0.61$ \\
\hline \multicolumn{5}{|l|}{ Smoking (\%) } \\
\hline Never & 89.3 & 84.6 & 88.9 & 85.2 \\
\hline Former & 3.6 & 11.5 & 7.4 & 3.7 \\
\hline Current & 7.1 & 3.8 & 3.7 & 11.1 \\
\hline Early cataracts $(\%)^{*}$ & 21.4 & 23.0 & 18.5 & 29.6 \\
\hline
\end{tabular}

There were no significant between-group differences in any baseline demographic or clinical variable.

For continuous variables, all values are mean \pm SD. Comparisons among groups were carried out using analysis of variance for continuous variables or the $\chi^{2}$ test for categorical variables.

${ }^{*}$ Cataracts diagnosed and graded according to the Lens Opacities Classification System III.

BMI, body mass index; HDL, high-density lipoprotein; LDL, low-density lipoprotein. 
Table 2 Changes in macular pigment optical density during supplementation in patients with early age-related macular degeneration

\begin{tabular}{|c|c|c|c|c|c|c|c|}
\hline \multirow[b]{2}{*}{ Time } & \multirow[b]{2}{*}{ Placebo $(n=28)$} & \multirow[b]{2}{*}{$10 \mathrm{mg}$ lutein $(n=26)$} & \multirow[b]{2}{*}{$20 \mathrm{mg}$ lutein $(n=27)$} & \multirow[b]{2}{*}{ Lutein+zeaxanthin $(n=27)$} & \multicolumn{3}{|c|}{ p Value } \\
\hline & & & & & Group & Time & Timexgroup \\
\hline Baseline & $0.315 \pm 0.144$ & $0.307 \pm 0.142$ & $0.315 \pm 0.122$ & $0.320 \pm 0.118$ & 0.072 & $<0.001$ & 0.046 \\
\hline 24 weeks & $0.310 \pm 0.102$ & $0.353 \pm 0.125$ & $0.395 \pm 0.120$ ** & $0.356 \pm 0.150$ & & & \\
\hline 48 weeks & $0.316 \pm 0.110$ & $0.371 \pm 0.188$ & $0.424 \pm 0.142^{* * *}$ & $0.384 \pm 0.125^{*}$ & & & \\
\hline 2 years & $0.324 \pm 0.163$ & $0.442 \pm 0.127^{* * *}$ & $0.441 \pm 0.133^{* * *}$ & $0.383 \pm 0.149 *$ & & & \\
\hline
\end{tabular}

and the change in N1P1 response densities in rings 3-6 (all $\mathrm{p}>0.05)$.

In contrast with the significant changes in N1P1 response densities from baseline to 48 weeks, we only observed a tendency toward improvement in MRS from 48 weeks to 2 years (all $\mathrm{p}>0.05$ ). However, differences between the placebo group and active treatment groups in total MRS and MRS at different eccentricities were seen at 48 weeks, especially at $3^{\circ}$ eccentricity
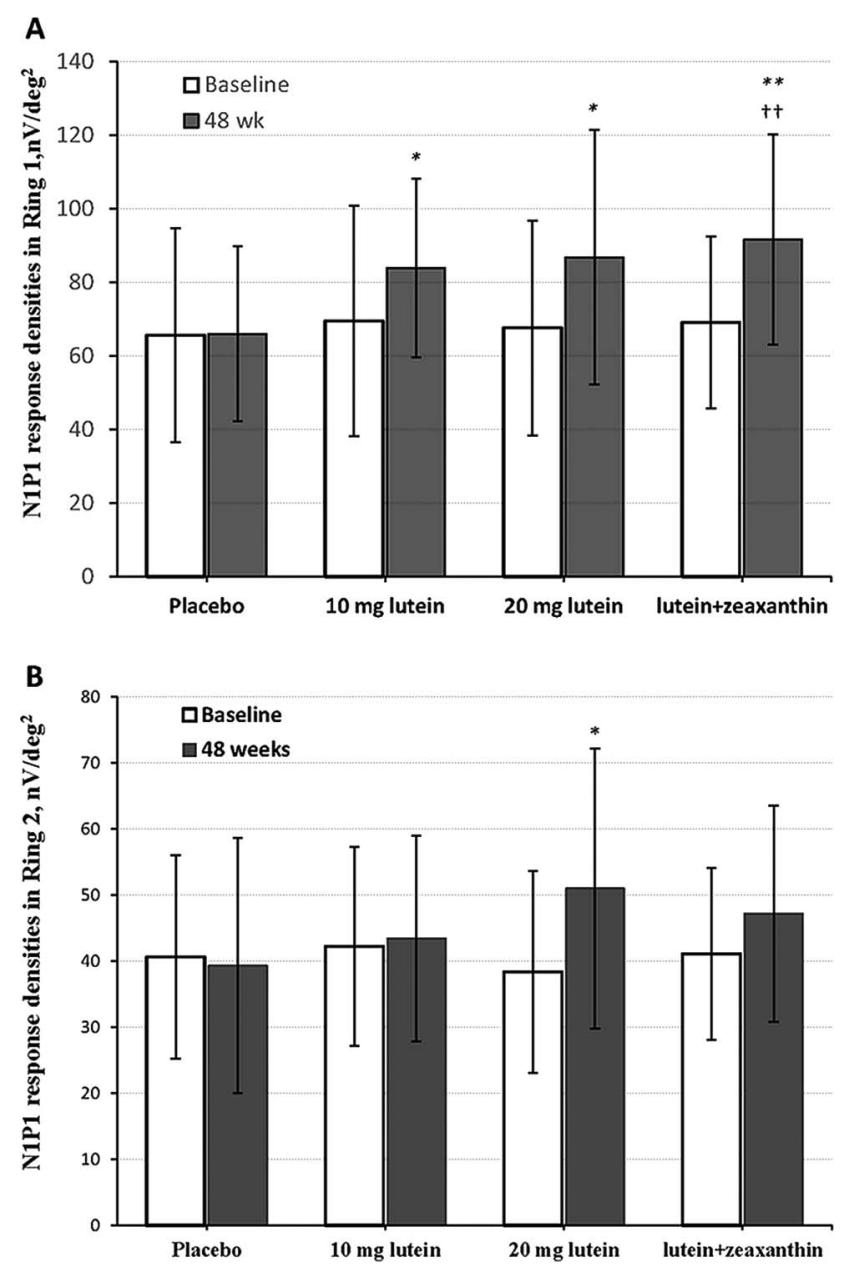

Figure 1 Bar graphs showing the change in N1P1 response densities in ring $1(A)$ and ring $2(B)$ at baseline and 48 weeks in patients with early age-related macular degeneration, treated with $10 \mathrm{mg} /$ day lutein, $20 \mathrm{mg} /$ day lutein, lutein (10 mg/day)+zeaxanthin (10 mg/day), or placebo. Values are expressed as group mean $\pm S D$. ${ }^{*} p<0.05,{ }^{* *} p<0.01$, significantly different from baseline within the same group (paired $t$ test). $t+p<0.01$, significantly different from placebo at each time point (repeated-measures analysis of variance with post hoc tests). $(\mathrm{p}<0.05)$ (figure $2 \mathrm{~A})$. At 2 years, in terms of MRS at $1^{\circ}$ eccentricity, significant differences between intervention groups and placebo $(10.32 \mathrm{~dB})$ were only observed for the $10 \mathrm{mg}$ $(13.37 \mathrm{~dB})$ and $20 \mathrm{mg}(12.55 \mathrm{~dB})$ lutein groups $(\mathrm{p}<0.05)$ (figure 2B).

\section{DISCUSSION}

In this randomised controlled study, 2 years of lutein/zeaxanthin intervention resulted in a significant MPOD increase, as well as functional retinal improvement in terms of a significant increase in N1P1 response densities and a tendency toward improvement in MRS in the central retina of patients with early AMD.

There is evidence that low MP might be a risk factor for $\mathrm{AMD}$, and the increase in MPOD following supplementation with macular carotenoids could benefit macular function in patients with AMD. ${ }^{8}$ The significant rise and the similar level of MPOD after supplementation with $10 \mathrm{mg}$ lutein and $20 \mathrm{mg}$ lutein at 2 years is not counterintuitive given the known distribution and saturable incorporation of the macular carotenoids in the retina. 8918

Since macular carotenoids accumulate in the central retina and decline dramatically with increasing retinal eccentricity, ${ }^{7}$ it may not be surprising that our supplementation mainly affected retinal function in the central area. Similar to other studies, our research resulted in a significant increase in N1P1 response densities in the central retina (ring 1 and ring 2) after 48 weeks of intervention. ${ }^{13}{ }^{19} \mathrm{~N} 1 \mathrm{P} 1$ response densities in ring 1 after supplementation with $20 \mathrm{mg}$ lutein $\left(86.8 \mathrm{nV} / \mathrm{deg}^{2}\right)$ and lutein +zeaxanthin $\left(91.6 \mathrm{nV} / \mathrm{deg}^{2}\right)$ increased to a similar level to that in non-diseased controls in the study of $\mathrm{Ma}$ et $a l^{13}(85.7 \mathrm{nV} /$ $\mathrm{deg}^{2}$ ). The highest level seen in the lutein+zeaxanthin group might be explained by some of the retina-captured lutein isomerising to meso-zeaxanthin in the central retina during the period of supplementation and therefore increasing all three macular carotenoids in the retina to maximally exert the antioxidant effects of $\mathrm{MP}^{20}{ }^{21}$ This rationale is also consistent with previous reports, both in non-diseased and AMD eyes, showing that supplementation with all three macular carotenoids offered more advantages in terms of MPOD response and visual performance. 92223

Given the above findings at 48 weeks, we introduced the MP-1 test, since it might provide additional information on improvement of retinal function when no more changes can be detected from other visual tests. ${ }^{24}$ We found significant differences in MRS in active treatment groups compared with the placebo group at 48 weeks, and a tendency toward improvement during the second year, indicating a possible continuous improvement in retinal sensitivities during the intervention. At 2 years, the most significant difference in MRS compared with placebo was observed at $1^{\circ}$ eccentricity after supplementation with $10 \mathrm{mg}$ lutein. This may be related to the continuous 
Figure 2 Bar graphs showing the change in mean retinal sensitivity (MRS) among groups at 48 weeks (A) and 2 years (B) in patients with early age-related macular degeneration, treated with $10 \mathrm{mg} /$ day lutein, $20 \mathrm{mg} /$ day lutein, lutein (10 mg/day)+zeaxanthin (10 mg/day) or placebo. Error bars indicate SE of the mean. $t p<0.05,+t p<0.01$ significantly different from placebo at each time point (repeated-measures analysis of variance with post hoc tests).

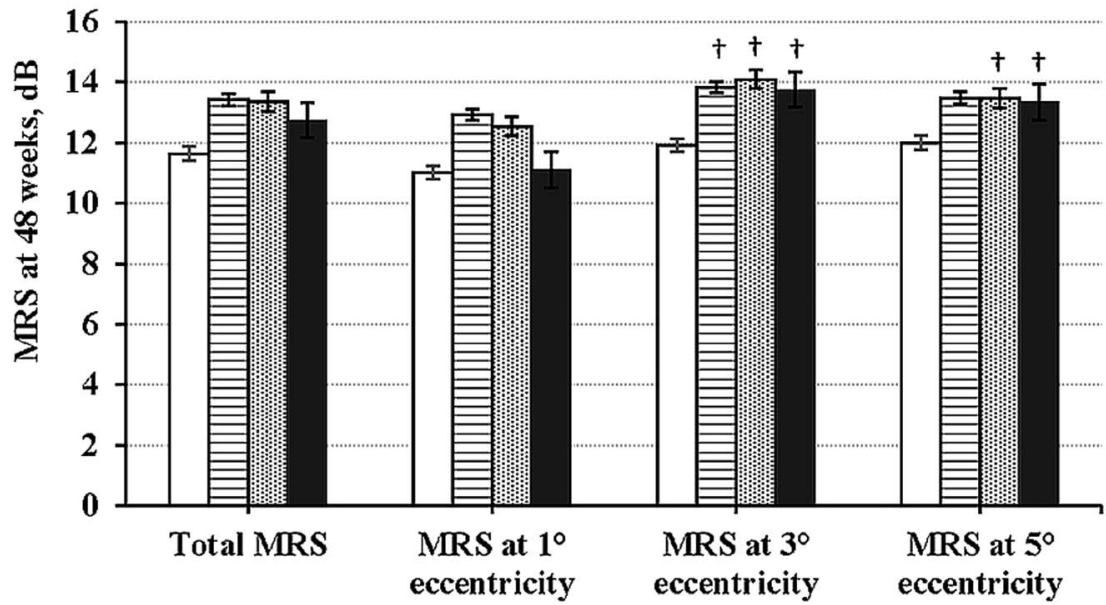

B $\square$ Placebo $610 \mathrm{mg}$ lutein 国20 $\mathrm{mg}$ lutein $\square$ lutein+zeaxanthin

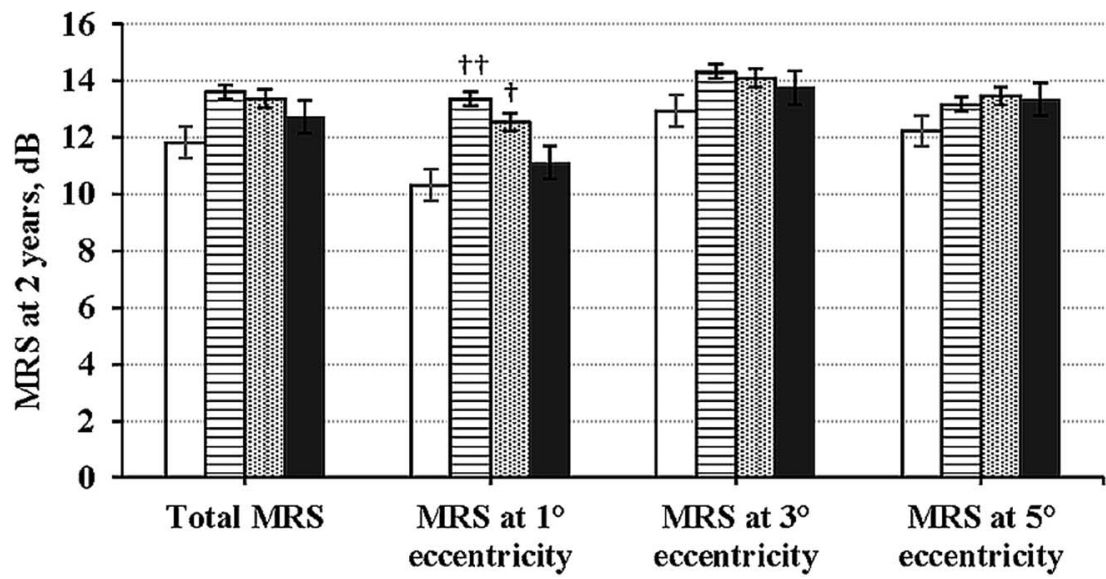

augmentation of MPOD in the $10 \mathrm{mg}$ lutein group during the same period. Our hypothesis is also supported by the significant association between MPOD and the changes in N1P1 response densities in the central retina seen in this study. All these correlations indicate that the change in MPOD is responsible for the change in retinal sensitivity, consistent with other studies. ${ }^{13} 19$ Supplementation with macular carotenoids might improve retinal function by increasing MP in patients with early AMD.

Improvement in retinal function as a result of MP augmentation is probably attributable to at least one of two mechanisms. First, prereceptoral filtration of blue light will attenuate the adverse effect of chromatic aberration and of veiling luminance on contrast sensitivity. ${ }^{25}$ Second, the antioxidant effect of macular carotenoids may exert a beneficial effect in maculae afflicted with conditions known to result from oxidative stress, such as AMD. ${ }^{21}$

There is a growing body of evidence that supplementation with macular carotenoids may also increase MPOD and visual performance in normal subjects without macular diseases. ${ }^{22}$ It is possible therefore that the implications of our findings may be clinically valuable for both AMD and normal subjects. In other words, MP augmentation is likely to enhance visual performance and experience in the elderly by attenuating the deleterious effects of chromatic aberration and veiling luminance on contrast sensitivity, irrespective of macular disease status (as long as the condition is not in its advanced form). Such optimisation of vision is clinically important for vulnerable older adults, as it will render daily activities, such as driving, easier and safer. ${ }^{22}$

There are limitations to this research. First, because of the highly selective criteria for subjects in this trial, our results may not be transferable to a broader population. Second, we did not include meso-zeaxanthin in our formula and did not have a zeaxanthin control group, which made it difficult to thoroughly discuss the effects of zeaxanthin and meso-zeaxanthin and their interactions with lutein supplementation. Third, our study cannot elucidate whether lutein/zeaxanthin supplementation is capable of reducing the progression of AMD, since neither the sample size nor the intervention period was sufficient to obtain such data. Further research is needed to evaluate the effect of macular carotenoids on progression of AMD.

In conclusion, this study has shown that MP can be augmented and visual function enhanced following supplementation with macular carotenoids in subjects with early AMD. The effect of MP augmentation on AMD progression warrants further investigation.

Acknowledgements We thank Xin Xiao, Xun Wang, Tingting Sun and Pengcheng Dong for helping with data collection, Le Ma for the first 48-week intervention, and Xin Wang, Fang Qian, Xin Wang and Honglei Pang for their reliable performance in ophthalmic examinations. A total of 114 subjects participated in the study, and we are grateful to them for their regular attendance. 
Contributors $\mathrm{Y}$-MH was responsible for subject recruitment, data collection and analysis, and drafting of the manuscript. F-FH, X-RX, X-RL and Z-YZ conducted the subject recruitment and data collection. X-ML and H-LD were the principal investigators and were responsible for the study design and supervision, interpretation of results, and critical review of the manuscript.

Funding Supported by the Chinese National Natural Science Foundation (grant 81273063)

Competing interests None.

Ethics approval Medical ethics committee of Peking University.

Provenance and peer review Not commissioned; externally peer reviewed.

\section{REFERENCES}

1 Ding $\mathrm{X}$, Patel M, Chan CC. Molecular pathology of age-related macular degeneration. Prog Retin Eye Res 2009;28:1-18.

2 Coleman HR, Chan CC, Ferris FR, et al. Age-related macular degeneration. Lancet 2008:372:1835-45.

3 Lim LS, Mitchell P, Seddon JM, et al. Age-related macular degeneration. Lancet 2012;379:1728-38.

4 Krinsky NI, Landrum JT, Bone RA. Biologic mechanisms of the protective role of lutein and zeaxanthin in the eye. Annu Rev Nutr 2003;23:171-201.

5 Moeller SM, Parekh N, Tinker L, et al. Associations between intermediate age-related macular degeneration and lutein and zeaxanthin in the Carotenoids in Age-related Eye Disease Study (CAREDS): ancillary study of the Women's Health Initiative. Arch Ophthalmol 2006;124:1151-62.

6 Tan JS, Wang JJ, Flood V, et al. Dietary antioxidants and the long-term incidence of age-related macular degeneration: the Blue Mountains Eye Study. Ophthalmology 2008;115:334-41.

7 Richer SP, Stiles W, Graham-Hoffman K, et al. Randomized, double-blind, placebo-controlled study of zeaxanthin and visual function in patients with atrophic age-related macular degeneration: the Zeaxanthin and Visual Function Study (ZVF) FDA IND \#78, 973. Optometry 2011;82:667-80

8 Weigert G, Kaya S, Pemp B, et al. Effects of lutein supplementation on macular pigment optical density and visual acuity in patients with age-related macular degeneration. Invest Ophthalmol Vis Sci 2011;52:8174-8.

9 Sabour-Pickett S, Beatty S, Connolly E, et al. Supplementation with three different macular carotenoid formulations in patients with early age-related macular degeneration. Retina 2014;34:1757-66.

10 Murray IJ, Makridaki M, van der Veen RL, et al. Lutein supplementation over a one-year period in early AMD might have a mild beneficial effect on visual acuity: the CLEAR study. Invest Ophthalmol Vis Sci 2013;54:1781-8.

11 The Age-Related Eye Disease Study 2 (AREDS2) Research Group. Lutein+zeaxanthin and omega-3 fatty acids for age-related macular degeneration: the Age-Related
Eye Disease Study 2 (AREDS2) randomized clinical trial. JAMA 2013;309: 2005-15.

12 Klein $\mathrm{R}$, Klein BE, Jensen SC, et al. The five-year incidence and progression of age-related maculopathy: the Beaver Dam Eye Study. Ophthalmology 1997;104:7-21.

13 Ma L, Dou HL, Huang YM, et al. Improvement of retinal function in early age-related macular degeneration after lutein and zeaxanthin supplementation: a randomized, double-masked, placebo-controlled trial. Am J Ophthalmol 2012;154:625-34.

14 Woo JH, Sanjay S, Au EK. Benefits of early awareness in age-related macular degeneration. Eye (Lond) 2009;23:2271; author reply 2271-2.

15 Davis MD, Gangnon RE, Lee LY, et al. The Age-Related Eye Disease Study severity scale for age-related macular degeneration: AREDS Report No. 17. Arch Ophthalmol 2005; 123:1484-98.

16 Ma L, Yan SF, Huang YM, et al. Effect of lutein and zeaxanthin on macular pigment and visual function in patients with early age-related macular degeneration. Ophthalmology 2012;119:2290-7.

17 Richer S, Stiles W, Statkute L, et al. Double-masked, placebo-controlled, randomized trial of lutein and antioxidant supplementation in the intervention of atrophic age-related macular degeneration: the Veterans LAST study (Lutein Antioxidant Supplementation Trial). Optometry 2004;75:216-30.

18 Trieschmann M, Beatty S, Nolan JM, et al. Changes in macular pigment optical density and serum concentrations of its constituent carotenoids following supplemental lutein and zeaxanthin: the LUNA study. Exp Eye Res 2007;84:718-28

19 Parisi V, Tedeschi M, Gallinaro G, et al. Carotenoids and antioxidants in age-related maculopathy Italian study: multifocal electroretinogram modifications after 1 year. Ophthalmology 2008;115:324-33.

20 Johnson EJ, Neuringer M, Russell RM, et al. Nutritional manipulation of primate retinas, III: Effects of lutein or zeaxanthin supplementation on adipose tissue and retina of xanthophyll-free monkeys. Invest Ophthalmol Vis Sci 2005;46: 692-702.

21 Li B, Ahmed F, Bernstein PS. Studies on the singlet oxygen scavenging mechanism of human macular pigment. Arch Biochem Biophys 2010;504:56-60.

22 Loughman J, Nolan JM, Howard AN, et al. The impact of macular pigment augmentation on visual performance using different carotenoid formulations. Invest Ophthalmol Vis Sci 2012;53:7871-80.

23 Connolly EE, Beatty S, Thurnham DI, et al. Augmentation of macular pigment following supplementation with all three macular carotenoids: an exploratory study. Curr Eye Res 2010;35:335-51.

24 Parravano M, Oddone $\mathrm{F}$, Tedeschi $\mathrm{M}$, et al. Retinal functional changes measured by microperimetry in neovascular age-related macular degeneration treated with ranibizumab: 24-month results. Retina 2010;30:1017-24.

25 Wooten BR, Hammond BR. Macular pigment: influences on visual acuity and visibility. Prog Retin Eye Res 2002;21:225-40. 